\title{
Intramolecular charge ordering in the multi molecular orbital system (TTM-TTP)I ${ }_{3}$
}

\author{
Marie-Laure Bonnet, ${ }^{1}$ Vincent Robert, ${ }^{1}$ Masahisa Tsuchiizu, ${ }^{2}$ Yukiko Omori, ${ }^{2}$ and Yoshikazu Suzumura ${ }^{2}$ \\ ${ }^{1}$ Laboratoire de Chimie, Ecole Normale Supérieure de Lyon, CNRS, 46 allée d'Italie, F-69364 Lyon, France \\ 2 Department of Physics, Nagoya University, Nagoya 464-8602, Japan
}

(Dated: June 8, 2010: J. Chem. Phys. 132, 214705 (2010))

\begin{abstract}
Starting from the structure of the (TTM-TTP) $\mathrm{I}_{3}$ molecular-based material, we examine the characteristics of frontier molecular orbitals using ab initio (CASSCF/CASPT2) configurations interaction calculations. It is shown that the singly-occupied and second-highest-occupied molecular orbitals are close to each other, i.e., this compound should be regarded as a two-orbital system. By dividing virtually the [TTM-TTP] molecule into three fragments, an effective model is constructed to rationalize the origin of this picture. In order to investigate the low-temperature symmetry breaking experimentally observed in the crystal, the electronic distribution in a pair of [TTM-TTP] molecules is analyzed from CASPT2 calculations. Our inspection supports and explains the speculated intramolecular charge ordering which is likely to give rise to low-energy magnetic properties.
\end{abstract}

\section{INTRODUCTION}

The characterization of molecule-based electronic conductors is one of the central issue in the research of molecular crystal systems. Typical materials are TTF-TCNQ and (TMTSF) ${ }_{2} \mathrm{PF}_{6}$, where TTF, TCNQ, and TMTSF stand for tetrathiafulvalene, tetracyanoquinodimethane, and tetramethyltetraselenafulvalene, respectively. In (TMTSF $)_{2} \mathrm{PF}_{6}$, the first superconductivity behavior in molecular solids has been reported. $\frac{1}{-}$ Recent hot topics in the research of molecular crystals is the realization of charge ordering phenomena. ${ }^{2}$ Since the pioneer work of $\mathrm{Su}$ et $a l^{\frac{3}{3}}$ in polyacetylene, the charge trapping phenomenon has been much studied in Peierls transition issues. ${ }^{4}$ In that sense, quasi-one-dimensional chains have received much attention from experimental and theoretical points of view $5-\frac{5}{2}$ Theoretical approaches that focus on a single highest-occupied-molecular orbital (HOMO) or lowestunoccupied-molecular orbital (LUMO) have been successful in describing fascinating electronic ordered phase. Such a treatment can be justified since in these conventional systems, the HOMO or LUMO levels are well-separated from the rest of the MOs spectrum. ${ }^{2}$

Nevertheless, new types of molecular solids have been recently synthesized, for instance, aiming at the metalization of the single-component molecular solids family $M(\mathrm{tmdt})_{2}$ $(M=\mathrm{Ni}, \mathrm{Au}), \frac{9}{9}$ where tmdt stands for trimethylenetetrathiafulvalenedithiolate. The molecular extension of the tmdt system is so large that a description based on a single molecular orbital (MO) is questionable. As a matter of fact, $a b$ initio calculations have been performed ${ }^{10-12}$ and an effective three-band Hubbard model has been proposed and succeeded in describing electronic structures. ${ }^{13}$ In this respect, the quasi-one-dimensional molecular compound (TTMTTP) $\mathrm{I}_{3}$, 14-16 where TTM-TTP=2,5-bis(4,5-bis(methylthio)1,3-dithiol-2-ylidene)-1,3,4,6-tetrathiapentalene looks like a promising candidate to investigate the theory limitation of a single-MO approximation. The presence of an $\mathrm{I}_{3}^{-}$counteranion is suggestive of formally organic [TTM-TTP $]^{+}$cations in the crystal structure, i.e., unpaired electrons localized within the organic moieties. The magnetic susceptibility ${ }^{17,18}$ and NMR measurements ${ }^{19,20}$ revealed phase transitions at finite temperature. Insulating and non-magnetic behaviors have been confirmed at low-temperature. Furthermore, based on
Raman-scattering ${ }^{21-23}$ and x-ray measurements, ${ }^{24}$ it has been suggested that an asymmetric deformation of the [TTM-TTP] molecule occurs and charge disproportionation within the molecule is possible below a transition temperature. This novel charge-ordered (CO) state is different from the conventional $\mathrm{CO}$ state $^{2}$ and is called intramolecular $\mathrm{CO}$ state. ${ }^{23}$ This nontrivial phenomena observed in this particular compound may not be described using a single-band description, i.e., the present molecular assembly could give rise to a phenomenon beyond a concept expected from a knowledge of a single molecule although the trigger may be hidden in the properties of multi molecular orbitals. Thus, there is a crucial need for theoretical description to rationalize the origin of such state. The purposes of our study are (i) to show that the energy of singly occupied molecular orbital (SOMO) and that of second-highest-occupied molecular orbital (HOMO1) are quasidegenerate, (ii) to clarify the origin of this quasidegeneracy using an effective three-fragment model for the [TTM-TTP] molecule, and (iii) to show explicitly that the intramolecular charge ordering actually occurs in the neighboring two-molecular system. With this goal in mind, multireference wave function-based $a b$ initio calculations are performed to rationalize the electronic distribution in the (TTM-TTP) $\mathrm{I}_{3}$ material.

\section{MOS OF THE [TTM-TTP $]^{+}$ION}

The [TTM-TTP] organic molecule $\mathrm{C}_{14} \mathrm{~S}_{12} \mathrm{H}_{12}$ is shown in Fig. 11 The atomic coordinates are read from the (TTMTTP) I $_{3} 298 \mathrm{~K}$ crystal structure ${ }^{15}$ In order to assess the packing influence in the electron trapping phenomenon, we did not perform any geometry optimization. Based on this structure, we performed correlated ab initio calculations. This type of approach is very insightful since important information is accessible through a reading of the wave function. In particular, complete active space self-consistent field (CASSCF) calculations have turned out to be very efficient to unravel intriguing electronic distribution in organic radical-based materials. ${ }^{25}-28$ On top of the CASSCF wave functions, second-order perturbation theory calculations (CASPT2) were performed using an imaginary shift of 0.3 a.u. and an ionization potentialelectronic affinity (IPEA) shift of 0.0 a.u. The IPEA shift 


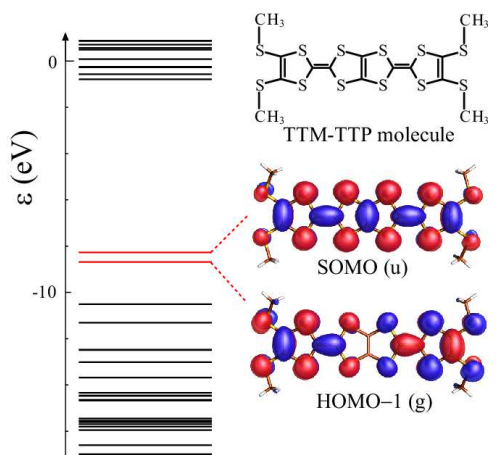

FIG. 1: [TTM-TTP] molecule (right-top) and energy levels of the isolated [TTM-TTP $]^{+}$ion from ROHF ab initio SCF calculations. The SOMO $(\mathrm{u})$ and HOMO-1 $(\mathrm{g})$ are drawn where the red and blue colors denote the sign of orbital.

aims at correcting the energy differences calculations between states holding different open shells. Since we are dealing with spin states, it would be irrelevant to turn on this parameter 29,30 This procedure allows one to incorporate the important dynamical correlation effects to reach a high level of accuracy. All our $a b$ initio calculations were performed using the MOLCAS package $\frac{31}{1}$ with all electron basis sets contractions for the elements $\mathrm{S}(7 \mathrm{~s} 6 \mathrm{p} 1 \mathrm{~d}) /[4 \mathrm{~s} 3 \mathrm{p} 1 \mathrm{~d}], \mathrm{C}(5 \mathrm{~s} 5 \mathrm{p} 1 \mathrm{~d}) /[3 \mathrm{~s} 2 \mathrm{p} 1 \mathrm{~d}]$ and $\mathrm{H}$ $(3 \mathrm{~s}) /[1 \mathrm{~s}]$. We checked the validity of these particular contractions by including diffuse and polarization functions which did not lead to any quantitative changes.

At room-temperature, the [TTM-TTP] molecule exhibits an inversion center. Thus, the MOs can be classified as gerade $(\mathrm{g})$ or ungerade (u) according to the symmetry point group. As mentioned before, the (TTM-TTP) $\mathrm{I}_{3}$ material is a charge-transfer salt, consisting of $[\mathrm{TTM}-\mathrm{TTP}]^{+}$and $\mathrm{I}_{3}^{-}$ species. In a simple picture, the HOMO of the [TTM-TTP] ${ }^{+}$ ion is half-filled, i.e., SOMO. It has been usually recognized that one may concentrate on this SOMO, ignoring the rest of the spectrum on the assumption that its energy is well isolated from those of the other orbitals as compared to the bandwidth. ${ }^{16}$ This is one particular issue we wanted to examine. Thus, semiempirical extended Hückel calculations were first performed ${ }^{32.33}$ In the following, the SOMO and HOMO1 will be referred to as the $\mathrm{u}$ and g orbitals, respectively (see Fig. (1). The energy separation between the $g$ and $u$ valence MOs (see Fig. 1) is $\approx 0.2 \mathrm{eV}$, while the bandwidth of the $\mathrm{HOMO}$ is $\approx 1 \mathrm{eV}: \underline{16}$ Thus, the effect of the g orbital might not be negligible since it is likely to participate in the intramolecular CO phenomenon expected in the (TTM-TTP) $\mathrm{I}_{3}$ system. In order to clarify the charge distribution, CASSCF calculations were then carried out allowing the occupation of two MOs by three electrons, i.e., CAS[3,2]. This method is known to provide very satisfactory charge distribution as soon as the active space is flexible enough. The $\mathrm{g}$ and $\mathrm{u}$ MOs are then treated on the same footing and both symmetries states can be examined along these calculations. The CASSCF energy difference between the $\mathrm{g}$ and $\mathrm{u}$ doublets is $\approx 0.5 \mathrm{eV}$, confirming the relative proximity of the frontier orbitals. The energy spectrum was finally calculated using a restricted open-shell a) $\mathrm{u} \mathrm{MO}$

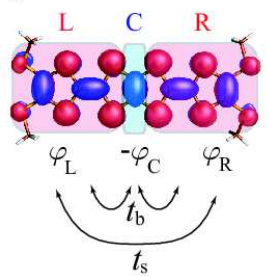

b) g MO

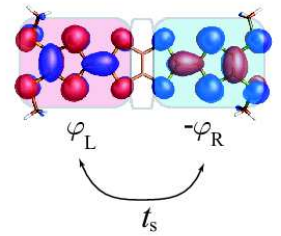

FIG. 2: Effective three-fragment model for the $\mathrm{u} \mathrm{MO}$ (a) and the $\mathrm{g}$ MO (b). The three fragments are named as L, R, and C, representing the left, right, and center fragments, and their wave functions are $\varphi_{\mathrm{L}}, \varphi_{\mathrm{R}}$, and $\varphi_{\mathrm{C}}$, respectively. The $\mathrm{u} \mathrm{MO}$ is given by the superposition of $\varphi_{\mathrm{L}},-\varphi_{\mathrm{C}}$, and $\varphi_{\mathrm{R}}$, while the $\mathrm{g} \mathrm{MO}$ is made of $\varphi_{\mathrm{L}}$ and $-\varphi_{\mathrm{R}}$. The mutual interaction between the respective fragments are $t_{\mathrm{b}}$ and $t_{\mathrm{s}}$, representing the through-bond and through-space interactions, respectively.

SCF (ROHF) procedure. Along these ROHF calculations, three electrons are likely to occupy the frontier MOs u and g. These MOs are likely to be singly occupied or doubly occupied in the CASSCF calculations. In order to position the corresponding energies, we performed a ROHF calculation assuming an average number of electrons in the $\mathrm{g}$ and $\mathrm{u}$ MOs, i.e., 1.5 electrons. The calculated energy levels in the vicinity of the SOMO are shown in Fig. 1 From this inspection, the SOMO has ungerade character, whereas the HOMO-1 is gerade type in agreement with our extended Hückel calculations. The respective ROHF energies $\varepsilon_{\mathrm{u}}=-8.27 \mathrm{eV}$ and $\varepsilon_{\mathrm{g}}=-8.69 \mathrm{eV}$, while the energy difference between the $\mathrm{u}$ and $\mathrm{g}$ MOs is $\approx 0.4 \mathrm{eV}$, a value which is consistent with our extended Hückel estimation. This combined semiempirical and ab initio information upon the constitutive unit [TTM-TTP $]^{+}$ raises the relevance of a one-band approach to examine the electronic properties of (TTM-TTP) $\mathrm{I}_{3}$ crystal.

\section{EFFECTIVE THREE-FRAGMENT MODEL}

In this section, we analyze the chemical origin of the closein-energy character of the $\mathrm{g}$ and $\mathrm{u}$ MOs. Let us split the [TTMTTP] molecule into 3 fragments $-\mathrm{L}, \mathrm{R}$, and $\mathrm{C}-$ as shown in Fig. 2. The $\mathrm{L}$ and $\mathrm{R}$ parts represent the left and right parts of the [TTM-TTP] molecule, whereas C corresponds to the ethylene-type bridging moiety. Based on this fragments picture, the u MO displays "bonding" character while the g MO is "anti-bonding". Therefore, one may wonder why the bonding MO lies higher in energy than the anti-bonding one. Part of the answer can be found in the $\mathrm{C}$ group orbital $\mathrm{u}$ which is likely to mix in the L-R "bonding" orbital (see Fig. 2). As for the interaction between these fragments, we consider two types of hopping integrals here, namely, $t_{\mathrm{b}}$ and $t_{\mathrm{s}}$. The former accounts for the through-bond interaction, while the latter represents the through-space interaction. The energy levels of the isolated $\mathrm{L}$ and $\mathrm{R}$ fragments are identical, and set to $\varepsilon_{0}$. On the other hand, the energy level of the isolated $\mathrm{C}$ fragment is much lower than the $\mathrm{L}$ and $\mathrm{R}$ fragments, and we parametrize the energy difference as $\Delta \varepsilon(>0)$. Using the local orbitals 
$\varphi_{\mathrm{L}}, \varphi_{\mathrm{R}}$, and $\varphi_{\mathrm{C}}$ shown in Fig. 2, the effective Hamiltonian for the 3-fragment model reads

$$
\left(\begin{array}{ccc}
\varepsilon_{0} & -t_{\mathrm{s}} & -t_{\mathrm{b}} \\
-t_{\mathrm{s}} & \varepsilon_{0} & -t_{\mathrm{b}} \\
-t_{\mathrm{b}} & -t_{\mathrm{b}} & \varepsilon_{0}-\Delta \varepsilon
\end{array}\right) \begin{aligned}
& \mathrm{L} \\
& \mathrm{R} \\
& \mathrm{C}
\end{aligned}
$$

First, let us neglect the through-bond $t_{\mathrm{b}}$ interaction. The through-space interaction between the $\mathrm{L}$ and $\mathrm{R}$ parts results in bonding and antibonding MOs whose energy difference is $2 t_{\mathrm{s}}$. This virtual situation is shown in the center of Fig. 3 As soon as the $t_{\mathrm{b}}$ interaction is turned on, the energy of the bonding MO is shifted higher, while the anti-bonding MO remain unchanged due to symmetry constraints. Assuming that $\Delta \varepsilon$ is much larger than $\left|t_{\mathrm{b}}\right|$, the eigenvalues read

$$
\begin{aligned}
\varepsilon_{\mathrm{u}} & \approx \varepsilon_{0}-t_{\mathrm{s}}+\frac{2 t_{\mathrm{b}}^{2}}{\Delta \varepsilon}, \\
\varepsilon_{\mathrm{g}} & =\varepsilon_{0}+t_{\mathrm{s}}, \\
\varepsilon_{\mathrm{u}^{\prime}} & \approx \varepsilon_{0}-\Delta \varepsilon-\frac{2 t_{\mathrm{b}}^{2}}{\Delta \varepsilon} .
\end{aligned}
$$

The respective wave functions are given by

$$
\begin{aligned}
\varphi_{\mathrm{u}} & =\frac{1}{\sqrt{2+a^{2}}}\left[\left(\varphi_{\mathrm{L}}+\varphi_{\mathrm{R}}\right)-a \varphi_{\mathrm{C}}\right] \\
\varphi_{\mathrm{g}} & =\frac{1}{\sqrt{2}}\left(\varphi_{\mathrm{L}}-\varphi_{\mathrm{R}}\right) \\
\varphi_{\mathrm{u}^{\prime}} & =\frac{-1}{\sqrt{1+a^{2} / 2}}\left[\varphi_{\mathrm{C}}+\frac{a}{2}\left(\varphi_{\mathrm{L}}+\varphi_{\mathrm{R}}\right)\right]
\end{aligned}
$$

where the quantity $a$ is given by $a \approx\left(2 t_{\mathrm{b}} / \Delta \varepsilon\right)$. In this picture, the $\mathrm{L}, \mathrm{R}$ and $\mathrm{C}$ fragments wave functions are assumed to be orthogonal which is obviously not a limitation for the description. By comparing Eq. (3) with the $u$ and g MOs shown in Fig. 11 one can conclude distinctly that the wave functions $\varphi_{\mathrm{u}}$ and $\varphi_{\mathrm{g}}$ correspond to the $\mathrm{u}$ and $\mathrm{g}$ MOs, respectively. To scope out the $\varphi_{\mathrm{u}^{\prime}}$ MO which is given in Eq. (3c),

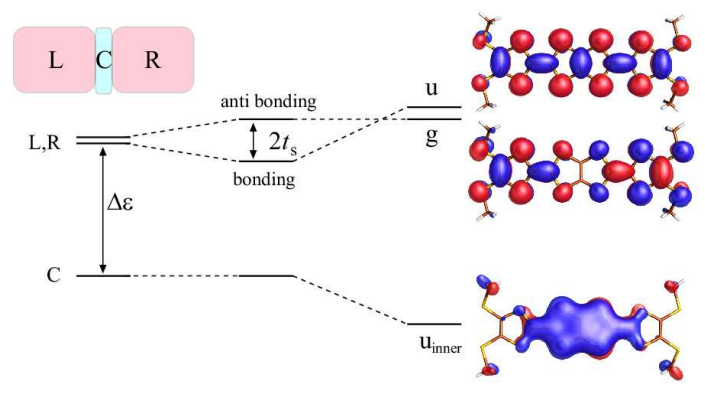

FIG. 3: MOs diagram for the effective three-fragment model of the [TTM-TTP] molecule. As the through-space interaction $t_{\mathrm{s}}$ is turned on, the $\mathrm{L}$ and $\mathrm{R}$ local orbitals mix in and result in bonding and antibonding MOs (center). Then, the through-bond interaction $t_{\mathrm{b}}$ pushes the bonding MOs higher in energy (right). The identified MOs, u, g, and, $\mathrm{u}_{\text {inner }}$, which are obtained from the SCF calculations, are also shown. The respective eigen-energies are given by $\varepsilon_{\mathrm{u}}=-8.27 \mathrm{eV}$, $\varepsilon_{\mathrm{g}}=-8.69 \mathrm{eV}$, and $\varepsilon_{\mathrm{u}_{\mathrm{inner}}}=-19.59 \mathrm{eV}$.

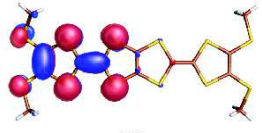

$\varphi_{\mathrm{L}}$

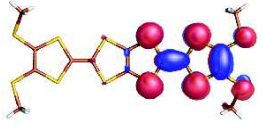

$\varphi_{\mathrm{R}}$
FIG. 4: The L and R MOs obtained through a unitary transformation of Eq. (3) by taking $a=0.25$.

we look into the set of ungerade MOs obtained from the SCF calculations. The in-phase combination $\mathrm{u}_{\text {inner }}$ of $\varphi_{\mathrm{L}}, \varphi_{\mathrm{R}}$, and $\varphi_{\mathrm{C}}$ wave functions is shown in Fig 3 and its energy is $\varepsilon_{\mathrm{u}_{\text {inner }}}=-19.59 \mathrm{eV}$. Since a typical value for $\left|t_{\mathrm{b}}\right|$ is of the order of several $\mathrm{eV}$, the latter energy separation fully justifies the approximate expressions given previously. Thus, we can assign the $\varphi_{\mathrm{u}^{\prime}}$ wave function to the $\mathrm{u}_{\mathrm{inner}}$ MO identified in the SCF calculations.

The ratio $2 t_{\mathrm{b}} / \Delta \varepsilon(\approx a)$ was estimated from the charge distribution in the [TTM-TTP] ${ }^{+}$ion, i.e., so-called Mulliken charges. Based upon the CAS[3,2] wave functions, CASPT2 calculations were performed and the resulting Mulliken charges were estimated. Following the three-fragment model, we summed up the Mulliken charges within each fragment, e.g., $\rho_{\mathrm{L}}=\sum_{i \in \mathrm{L}} \rho_{i}$ where $\rho_{i}$ is the charge of the respective atoms. In the ground state configuration where the $\mathrm{u}$ MO is singly occupied, the Mulliken charges on the respective fragments of the [TTM-TTP] ${ }^{+}$ion were estimated as

$$
\rho_{\mathrm{L}}=\rho_{\mathrm{R}}=+0.634, \quad \rho_{\mathrm{C}}=-0.268 .
$$

The total charge is $\rho_{\mathrm{L}}+\rho_{\mathrm{R}}+\rho_{\mathrm{C}}=+1$. In order to extract the contribution from the SOMO, we analyzed the charge difference between the cation [TTM-TTP $]^{+}$and the [TTMTTP] molecule. The latter exhibits the following Mulliken charges on the respective fragments $\rho_{\mathrm{L}}^{0}=\rho_{\mathrm{R}}^{0}=+0.149$ and $\rho_{\mathrm{C}}^{0}=-0.297$. Thus, the charge differences $\Delta \rho_{Z} \equiv\left(\rho_{Z}-\rho_{Z}^{0}\right)$ $(Z=\mathrm{L}, \mathrm{R}, \mathrm{C})$ were calculated as

$$
\Delta \rho_{\mathrm{L}}=\Delta \rho_{\mathrm{R}}=0.485, \quad \Delta \rho_{\mathrm{C}}=0.029 .
$$

This means that the hole is mainly localized on the $\mathrm{L}$ and $\mathrm{R}$ fragments, whereas the charge on the $\mathrm{C}$ fragment remains almost unchanged. By combining the information upon the SOMO [Eq. (3a)] and the numerical data of the charge distribution [Eq. (5)], we can estimate the parameter $a \approx\left(2 t_{\mathrm{b}} / \Delta \varepsilon\right)$ by using the relations $1 /\left(2+a^{2}\right)=\Delta \rho_{\mathrm{L}}$ or $a^{2} /\left(2+a^{2}\right)=$ $\Delta \rho_{\mathrm{C}}$. Based on this analysis, we find $a \approx 0.25$ and finally the ratio $\left(t_{\mathrm{b}} / \Delta \varepsilon\right)$ is $\approx 0.12$. Once the parameter $a$ is determined, the fragment wave functions can be obtained explicitly through the inverse unitary transformation of Eq. (3). The resulting $\mathrm{L}$ and $\mathrm{R}$ MOs are shown in Fig. 4 and correspond to the $\varphi_{\mathrm{L}}$ and $\varphi_{\mathrm{R}}$ local MOs. Since $\left[\left(\varepsilon_{\mathrm{u}}+\varepsilon_{\mathrm{g}}\right) / 2-\varepsilon_{\mathrm{u}_{\text {inner }}}\right]=$ $\Delta \varepsilon\left[1+3\left(t_{\mathrm{b}} / \Delta \varepsilon\right)^{2}\right]$ [see Eq. (2)], the ROHF eigenvalues $\varepsilon_{\mathrm{u}}=-8.27 \mathrm{eV}, \varepsilon_{\mathrm{g}}=-8.69 \mathrm{eV}$, and $\varepsilon_{\mathrm{u}_{\mathrm{inner}}}=-19.59 \mathrm{eV}$ lead to the effective energy of the $\mathrm{C}$ fragment $\Delta \varepsilon \approx 10.6 \mathrm{eV}$. Finally, the magnitudes of the through-bond/through-space interactions can be estimated, $t_{\mathrm{b}} \approx 1.32 \mathrm{eV}$ and $t_{\mathrm{s}} \approx-0.04 \mathrm{eV}$. From our evaluation, $t_{\mathrm{s}}$ is almost negligible, and the bonding and antibonding MOs of Fig. 3 are almost degenerated. The 
through-space integral is much smaller than the through-bond one, $\left|t_{\mathrm{b}} / t_{\mathrm{s}}\right| \approx 28$. Since the energy difference between the $\mathrm{u}$ and $\mathrm{g}$ MOs is determined from Eq. (2)

$$
\varepsilon_{\mathrm{u}}-\varepsilon_{\mathrm{g}} \approx-2 t_{\mathrm{s}}+\frac{2 t_{\mathrm{b}}^{2}}{\Delta \varepsilon}
$$

the origin of the quasidegeneracy of the $u$ and g MOs can be clarified in the light of the extracted parameters.

In conclusion, the $\mathrm{u}$ and $\mathrm{g}$ MOs ordering is completely determined by the relative energetics of this three-piece molecule. The through-space interaction $t_{\mathrm{s}}$ is almost negligible, while through-bond interaction $t_{\mathrm{b}}$ determines the energy spectrum.

\section{INTRAMOLECULAR CHARGE ORDERING}

In this section, the interactions between neighboring [TTM$\mathrm{TTP}^{+}$cations have been investigated using a dimer extracted from the (TTM-TTP) $\mathrm{I}_{3}$ crystal. We used the atomic coordinates of the crystal structure at $298 \mathrm{~K}$. If the crystal-structure data at low-temperature phase were available, we may perform more quantitative analysis by combining the geometrical optimization. However, it can be considered that the following results are not affected qualitatively. Since we were not only interested in charge distribution, CASPT2 calculations were also performed to specify the low-energy spectroscopy of the $[\text { TTM-TTP }]_{2}^{2+}$ dimer.

\section{A. "MOs"}

First, CASSCF calculations were carried out on the [TTM$\mathrm{TTP}_{2}^{2+}$ dimer. These calculations were performed including six electrons in four MOs in the active space (CAS[6,4]) to account for the important static correlation effects. The interactions between the neighboring [TTM-TTP $]^{+}$ions labeled as I and II give rise to the effective MOs shown in Fig. 5. Importantly, the dimer exhibits an inversion center and the resulting MOs can be classified into gerade and ungerade. Nevertheless, the inversion center of each individual subunit is lost, which might lead to electron localization within the [TTM$\mathrm{TTP}]^{+}$building blocks. As expected, the CAS[6,4]SCF frontier orbitals (Fig. 5) consist in the in-phase and out-of-phase combinations of the $\varphi_{\mathrm{L}, \mathrm{I}}, \varphi_{\mathrm{R}, \mathrm{I}}, \varphi_{\mathrm{L}, \mathrm{II}}$, and $\varphi_{\mathrm{R}, \mathrm{II}}$ MOs. The $\varphi_{\mathrm{L}, \mathrm{I}}$ and $\varphi_{\mathrm{R}, \mathrm{I}}$ are the left (L) and right (R) localized MOs on the subunit I (see Fig. 4). A similar definition holds for the subunit II.

From the subsequent CASPT2 treatment, the ground state is a gerade singlet, and the active MOs exhibit occupation numbers $2.0,2.0,0.9$, and 1.1, respectively. Importantly, the occupation numbers of the $\mathrm{g}_{2}$ and $\mathrm{u}_{2}$ MOs strongly deviate from 2 and 0 , a feature of the open-shell nature of the ground state singlet. The symmetry breaking within each [TTM-TTP $]^{+}$ ion induced by the presence of a second partner leads to a mixing of the orbitals depicted in Fig. 3] This particular mechanism is likely to result in the charge ordering we now wish to examine.

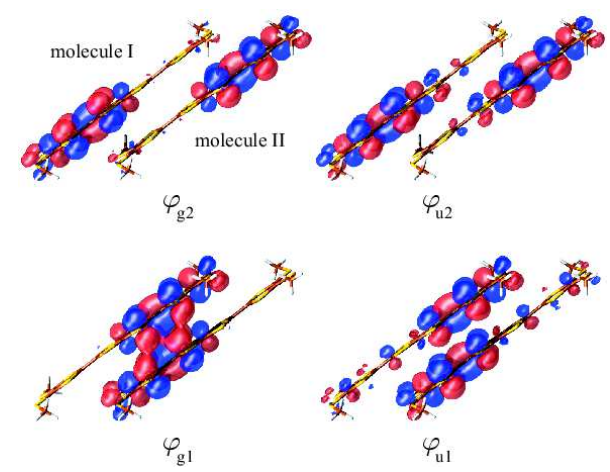

FIG. 5: Valence MOs of the $[\text { TTM-TTP }]_{2}^{2+}$ system obtained from CAS[6,4]SCF ab initio calculations upon the singlet state of g symmetry.

\section{B. Mulliken charge}

From the CASPT2 calculations performed upon the [TTM$\mathrm{TTP}]_{2}^{2+}$ dimer, a similar Mulliken charge analysis was carried out to quantify the charge redistribution accompanying the dimer formation. The ground singlet state exhibits the following charge reorganization as compared to the isolated neutral [TTM-TTP] molecule

$$
\Delta \rho_{\mathrm{L}}=0.697, \quad \Delta \rho_{\mathrm{R}}=0.272, \quad \Delta \rho_{\mathrm{C}}=0.030 .
$$

As a major conclusion, the charge on the left (right) fragment is strongly enhanced (reduced), which is a clear indication of the intramolecular charge ordering. Again, the charge on the center fragment $\mathrm{C}$ is almost unchanged. The present evaluation based on the two-molecule dimer overestimates the charge difference between the $\mathrm{L}$ and $\mathrm{R}$ fragments as compared to its value in the crystal, and also the validity of Mulliken charges remains questionable since the fluctuation effects were not included. Nevertheless, this qualitative analysis supports the intramolecular CO state.

\section{Low-energy spectroscopy}

Our Mulliken charge analysis is suggestive of an intramolecular charge ordering mechanism in the (TTM-TTP) $\mathrm{I}_{3}$ material. Thus, one may expect a charge localization on the left or right parts of the TTM-TTP building blocks. Such scenario is likely to give rise to magnetic interactions involving either the inner parts (i.e., $\varphi_{\mathrm{R}, \mathrm{I}}$ and $\varphi_{\mathrm{L}, \mathrm{II}}$ fragments MOs) or the outer parts (i.e., $\varphi_{\mathrm{L}, \mathrm{I}}$ and $\varphi_{\mathrm{R}, \mathrm{II}}$ ).

Thus, starting from the CAS[6,4]SCF calculations, the lowenergy spectroscopy of the $[\text { TTM-TTP }]_{2}^{2+}$ species was inspected. The multi-reference CASSCF wave functions were expanded using a local orbitals basis set $\left\{\varphi_{\mathrm{L}, \mathrm{I}}, \varphi_{\mathrm{R}, \mathrm{I}}, \varphi_{\mathrm{L}, \mathrm{II}}\right.$, $\left.\varphi_{\mathrm{R}, \mathrm{II}}\right\}$ following the transformation

$$
\begin{aligned}
& \varphi_{\mathrm{g} 1} \approx \frac{1}{\sqrt{2}}\left(-\varphi_{\mathrm{R}, \mathrm{I}}+\varphi_{\mathrm{L}, \mathrm{II}}\right), \\
& \varphi_{\mathrm{u} 1} \approx \frac{1}{\sqrt{2}}\left(-\varphi_{\mathrm{R}, \mathrm{I}}-\varphi_{\mathrm{L}, \mathrm{II}}\right),
\end{aligned}
$$


TABLE I: Wave functions decompositions (weights) of the $[\text { TTM-TTP }]_{2}^{2+}$ low-energy states. The wave functions are expressed in terms of the two hole determinants. CAS[6,4]PT2 energies with respect to the ground state singlet $\mathrm{g}$ are given in $\mathrm{eV}$.

\begin{tabular}{|c|c|c|c|c|c|c|c|}
\hline & $\begin{array}{c}\left|\varphi_{\mathrm{L}, \mathrm{I}} \bar{\varphi}_{\mathrm{R}, \mathrm{II}}\right\rangle \\
(\%)\end{array}$ & $\begin{array}{c}\left|\varphi_{\mathrm{R}, \mathrm{I}} \bar{\varphi}_{\mathrm{L}, \mathrm{II}}\right\rangle \\
(\%)\end{array}$ & $\begin{array}{c}\left|\varphi_{\mathrm{L}, \mathrm{I}} \bar{\varphi}_{\mathrm{R}, \mathrm{I}}\right\rangle \\
(\%)\end{array}$ & $\begin{array}{c}\left|\varphi_{\mathrm{L}, \mathrm{I}} \bar{\varphi}_{\mathrm{L}, \mathrm{I}}\right\rangle \\
(\%)\end{array}$ & $\begin{array}{c}\left|\varphi_{\mathrm{L}, \mathrm{I}} \bar{\varphi}_{\mathrm{L}, \mathrm{I}}\right\rangle \\
(\%)\end{array}$ & $\begin{array}{c}\left|\varphi_{\mathrm{R}, \mathrm{I}} \bar{\varphi}_{\mathrm{R}, \mathrm{I}}\right\rangle \\
(\%)\end{array}$ & $\begin{array}{l}\text { Energy } \\
(\mathrm{eV})\end{array}$ \\
\hline \multirow[t]{3}{*}{ Singlet, gerade } & 84 & 1 & 3 & 9 & 3 & 0 & $E_{0}=0.00$ \\
\hline & 11 & 27 & 44 & 13 & 5 & 0 & $E_{2}=0.27$ \\
\hline & \multicolumn{2}{|c|}{$\begin{array}{c}\left|\varphi_{\mathrm{L}, \mathrm{I}} \varphi_{\mathrm{R}, \mathrm{II}}\right\rangle \\
(\%)\end{array}$} & $\begin{array}{c}\left|\varphi_{\mathrm{R}, \mathrm{I}} \varphi_{\mathrm{L}, \mathrm{II}}\right\rangle \\
(\%)\end{array}$ & \multicolumn{2}{|c|}{$\begin{array}{c}\left|\varphi_{\mathrm{L}, \mathrm{I}} \varphi_{\mathrm{R}, \mathrm{I}}\right\rangle \\
(\%)\end{array}$} & $\begin{array}{c}\left|\varphi_{\mathrm{L}, \mathrm{I}} \varphi_{\mathrm{L}, \mathrm{II}}\right\rangle \\
(\%)\end{array}$ & $\begin{array}{c}\text { Energy } \\
(\mathrm{eV})\end{array}$ \\
\hline \multirow[t]{3}{*}{ Triplet, ungerade } & \multicolumn{2}{|c|}{62} & 5 & \multicolumn{2}{|c|}{0} & 33 & $E_{1}=0.09$ \\
\hline & \multicolumn{2}{|r|}{28} & 47 & \multicolumn{2}{|c|}{2} & 23 & $E_{3}=0.47$ \\
\hline & \multicolumn{2}{|r|}{6} & 8 & \multicolumn{2}{|c|}{70} & 16 & $E_{4}=0.62$ \\
\hline
\end{tabular}

$$
\begin{aligned}
& \varphi_{\mathrm{g} 2} \approx \frac{1}{\sqrt{2}}\left(+\varphi_{\mathrm{L}, \mathrm{I}}-\varphi_{\mathrm{R}, \mathrm{II}}\right), \\
& \varphi_{\mathrm{u} 2} \approx \frac{1}{\sqrt{2}}\left(-\varphi_{\mathrm{L}, \mathrm{I}}-\varphi_{\mathrm{R}, \mathrm{II}}\right) .
\end{aligned}
$$

Since we are dealing with a six-electron/four-MO system, we performed our analysis in the two-hole/four-MO picture. Schematic representations of the different hole configurations for the singlet and triplet states based on these local orbitals are shown in Fig. 6. Such transformation affords a reading of the different wave functions and the extractions of the relevant information in a valence-bond type analysis. Since $\left|t_{s}\right|$ is relatively small, the left and right moieties within each unit do not significantly overlap. Thus, the previous transformation is almost unitary.

The lowest lying states of the $[\text { TTM-TTP }]_{2}^{2+}$ species are given in Table [I. The ground state is singlet $\mathrm{g}$ and consistent with a picture that minimizes the electrostatic energy between the two [TTM-TTP] ${ }^{+}$ions. The comparison between the ground state singlet $g$ and first triplet $u$ is very instructive. In both cases, the wave function is largely dominated by the electronic configurations (84\% and $62 \%$ in Table $\square$ for the singlet and triplet states, respectively) involving the inner parts (see Fig. 6). The energy difference affords an evaluation of the intermolecular magnetic exchange interaction :

$$
J \equiv E_{1}-E_{0} \approx 0.09 \mathrm{eV}
$$

A positive value reflects an antiferromagnetic interaction, re-

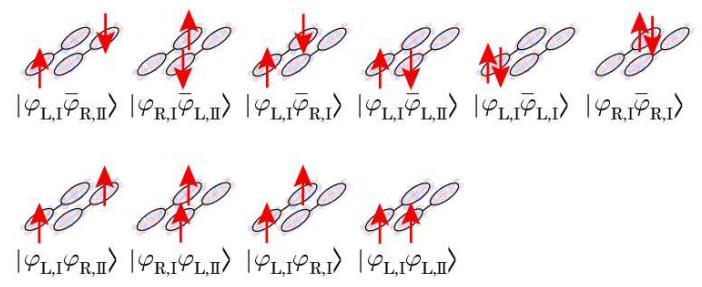

FIG. 6: Schematic view of the different configurations using a hole picture for the singlet (top) and triplet (bottom), respectively. The subscripts I and II stand for the molecules I and II as depicted in Fig. [5 sulting from the intramolecular charge ordering. Such a value suggests that the spin gap excitation energy should be $\approx 900$ $\mathrm{K}$. The first-excited singlet corresponds to the holes localization within one $[\text { TTM-TTP }]^{+}$ion. This is a reflection of an intermolecular charge ordering which lies much higher and might not be relevant to describe the low-energy properties of the (TTM-TTP) $\mathrm{I}_{3}$ material. The energy difference $(0.35 \mathrm{eV})$ between this second excited state (singlet $\mathrm{g}$ ) and the fourth one (triplet $\mathrm{u}$ ) supports the strong antiferromagnetic interaction within each subunit. This is a mechanism that involves again intermolecular electron transfer. The low-energy spectroscopy in the (TTM-TTP) $I_{3}$ material should be mostly governed by the through-space interdimer magnetic interaction $J$, which results from the intramolecular charge ordering.

\section{SUMMARY AND DISCUSSIONS}

In the present paper, we examined the low-energy properties of the (TTM-TTP) $I_{3}$ material. Complementary semiempirical and wave function-based ab initio calculations were performed upon the elementary unit and a dimer to investigate the underlying electronic distribution. We showed that the SOMO and HOMO- 1 are close in energy and that a oneband picture should be ruled out. A chemical understanding arises from the inspection of the building block and effective parameters extracted from a three-fragment model allowed us to rationalize the quasidegeneracy of the [TTM-TTP] frontier MOs. From the calculation of a system consisting of two neighboring molecules, the inversion symmetry within each molecule is lost. This result is in agreement with experimental data. Indeed, the intramolecular charge ordering which has been experimentally suggested actually occurs in the two-molecule cluster system. The mixing of the SOMO and HOMO-1 results in an electron trapping which results in a 0.697 / 0.272 on the L / R moieties of the [TTM-TTP] ${ }^{+}$ ion. Finally, our $a b$ initio calculations suggest that the lowenergy of the (TTM-TTP) $\mathrm{I}_{3}$ material is controlled by a single exchange interaction $0.09 \mathrm{eV}$, resulting from the charge ordering.

In order to complement our Mulliken charge analysis, we also performed preliminary Raman and infrared (IR) calcu- 
lations upon the $[\text { TTM-TTP }]^{+}$unit. It has been observed upon cooling that the Raman $1490 \mathrm{~cm}^{-1}$ band splits into two peaks centered at $1487 \mathrm{~cm}^{-1}$ and $1499 \mathrm{~cm}^{-1}$, where this phenomenon was attributed to the differentiation between the $\mathrm{C}=\mathrm{C}$ ylidene bonds, featuring a symmetry breaking. ${ }^{21} \mathrm{We}$ used the GAUSSIAN03 package ${ }^{34}$ on isolated one- and twomolecule systems, and full geometry optimizations were carried out on both systems. The information extracted from the one-molecule system should be compared with the hightemperature regime. In contrast, the two-molecule system is expected to give access to the low-temperature Raman spectrum characteristics. The calculated spectrum of twomolecule system displays two vibrational frequencies, 1522 $\mathrm{cm}^{-1}$ and $1534 \mathrm{~cm}^{-1}$, while that of the 1-molecule system shows a single band at $1535 \mathrm{~cm}^{-1}$. Despite a general blueshift, this result is in agreement with experimental data, and can be attributed to an intramolecular CO phase accompanying the descent in symmetry within the TTM-TTP units. In this calculation, the $[\mathrm{TTM}-\mathrm{TTP}]_{2}^{2+}$ dimer was extracted from the available crystallographic data. A direct comparison with experimental findings would rely on Raman and IR calculations using the low-temperature crystal structure. Unfortu- nately, the lack of such x-ray data disposes of this strategy.

Throughout this work, we have focused only on the [TTMTTP] ion and have neglected the effect of the counteranion of $\mathrm{I}_{3}^{-}$. As a matter of fact, it has been pointed out, from $\mathrm{X}$-ray measurement, $\frac{18}{}$ that the large displacement of the $\mathrm{I}_{3}$ species occurs in the low-temperature phase. This might yield changes in the electrostatic potential, and possibly the electronic distribution in the [TTM-TTP] ion would qualitatively be modified. This point is to be studied in future work.

\section{Acknowledgements}

MT thanks T. Kawamoto, T. Mori, H. Kobayashi, and S. Yasuzuka, for the fruitful discussions on the (TTM-TTP) $\mathrm{I}_{3}$ compounds. MLB, MT, YO, and YS thank S. Ishibashi and H. Seo for the discussions on the theoretical aspects. MT thanks $\mathrm{S}$. Yasuzuka for the enlightening discussions in the early stage of the present work. MLB was supported by the JSPS postdoctoral fellowship for Foreign Researchers. This research was partially supported by Nagoya University Science Foundation and Grant-in-Aid for Scientific Research on Innovative Areas (20110002) from the Ministry of Education, Culture, Sports, Science and Technology, Japan.
1 D. Jerome and H.J. Schulz, Adv. Phys. 31, 299 (1982).

${ }^{2}$ H. Seo, C. Hotta, and H. Fukuyama, Chem. Rev. 104, 5005 (2004).

3 W.P. Su, J.R. Shrieffer, and A.J. Heeger, Phys. Rev. Lett. 42, 1698 (1979).

4 R. Peierls, in Quantum Theory of Solids (Clarendon: Oxford, U.K. 1955).

5 S.A. Borshch, K. Prassides, V. Robert, and A.O. Solonenko, J. Chem. Phys. 109, 4562 (1998).

${ }^{6}$ V. Robert, S. Petit, and S.A. Borshch, Inorg. Chem. 38, 1573 (1999).

7 V. Robert, J. Chem. Phys. 121, 4297 (2004).

8 J.-P. Malrieu and V. Robert, J. Chem. Phys. 120, 7374 (2004).

${ }^{9}$ H. Tanaka, Y. Okano, H. Kobayashi, W. Suzuki, and A. Kobayashi, Science 291, 285 (2001).

${ }^{10}$ C. Rovira, J.J. Novoa, J.-L. Mozos, P. Ordejón, and E. Canadell, Phys. Rev. B 65 (2002) 081104.

${ }^{11}$ S. Ishibashi, H. Tanaka, M. Kohyama, M. Tokumoto, A. Kobayashi, H. Kobayashi, and K. Terakura, J. Phys. Soc. Jpn. 74, 843 (2005).

12 S. Ishibashi, K. Terakura, and A. Kobayashi, J. Phys. Soc. Jpn. 77 (2008) 024702.

${ }^{13}$ H. Seo, S. Ishibashi, Y. Okano, H. Kobayashi, A. Kobayashi, H. Fukuyama, and K. Terakura, J. Phys. Soc. Jpn. 77, 023714 (2008)

${ }^{14}$ For a review, T. Mori, Chem. Rev. 104, 4947 (2004).

15 T. Mori, H. Inokuchi, Y. Misaki, T. Yamabe, H. Mori, and S. Tanaka, Bull. Chem. Soc. Jpn. 67, 661 (1994).

16 T. Mori, T. Kawamoto, J. Yamaura, T. Enoki, Y. Misaki, T. Yamabe, H. Mori, and S. Tanaka, Phys. Rev. Lett. 79, 1702 (1997).

17 M. Maesato, Y. Sasou, S. Kagoshima, T. Mori, T. Kawamoto, Y. Misaki, and T. Yamabe, Synth. Met. 103, 2109 (1999).

${ }^{18}$ N. Fujimura, A. Namba, T. Kambe, Y. Nogami, K. Oshima, T. Mori, T. Kawamoto, Y. Misaki, and T. Yamabe, Synth. Met. 103, 2111 (1999).

19 M. Onuki, K. Hiraki, T. Takahashi, D. Jinno, T. Kawamoto, T.
Mori, T. Takano, and Y. Misaki, Synth. Met. 120, 921 (2001)

${ }^{20}$ M. Onuki, K. Hiraki, T. Takahashi, D. Jinno, T. Kawamoto, T. Mori, K. Tanaka, and Y. Misaki, J. Phys. Chem. Solids 62, 405 (2001).

${ }^{21}$ K. Yakushi, R. Świetlik, K. Yamamoto, T. Kawamoto, T. Mori, Y. Misaki, and K. Tanaka, Synth. Met. 135-136, 583 (2003).

${ }^{22}$ R. Świetlik, K. Yakushi, K. Yamamoto, T. Kawamoto, and T. Mori, J. Phys. IV France 114, 87 (2004).

${ }^{23}$ R. Świetlik, K. Yakushi, K. Yamamoto, T. Kawamoto, T. Mori, Synth. Met. 150, 83 (2005).

${ }^{24}$ Y. Nogami, T. Kambe, N. Fujimura, K. Oshima, T. Mori, and T. Kawamoto, Synth. Met. 135-136, 637 (2003).

25 J.-B. Rota, L. Norel, C. Train, N. Ben Amor, D. Maynau, and V. Robert, J. Am. Chem. Soc. 130, 10380 (2008).

26 S. Messaoudi, V. Robert, N. Guihery, and D. Maynau, Inorg. Chem. 45, 3212 (2006).

${ }^{27}$ M. Kepenekian, B. Le Guennic, K. Awaga, and V. Robert, Phys. Chem. Chem. Phys. 11, 6066 (2009).

28 J.-B. Rota, B. Le Guennic, and V. Robert, Inorg. Chem. 49, 1230 (2010).

${ }^{29}$ G. Ghigo, B.O. Roos, and P. Malmqvist, Chem. Phys. Lett. 396, 142 (2004).

${ }^{30}$ M. Kepenekian, V. Robert, and B. Le Guennic, J. Chem. Phys. 131, 114702 (2009).

${ }^{31}$ G. Karlström, R. Lindh, P.-Å. Malmqvist, B.O. Roos, U. Ryde, V. Veryazov, P.-O. Widmark, M. Cossi, B. Schimmelpfennig, P. Neogrady, and L. Seijo, Comput. Mater. Sci. 28 (2003) 222.

${ }^{32}$ T. Mori, A. Kobayashi, Y. Sasaki, H. Kobayashi, G. Saito, and H. Inokuchi, Bull. Chem. Soc. Jpn. 57, 627 (1984).

33 T. Mori, Doctor Thesis, Univ. Tokyo (1985), Chap. 4.

${ }^{34}$ M.J. Frisch G.W. Trucks, H.B. Schlegel et al., GAUSSIAN 03, Gaussian, Inc., Pittsburgh PA, 2003, we have used a BLYP functional and a triple zeta basis set. 